DESY 18-216, DO-TH 18/28

\title{
Large scale analytic calculations in quantum field theories
}

\author{
Johannes Blümlein
}

\begin{abstract}
We present a survey on the mathematical structure of zero- and single scale quantities and the associated calculation methods and function spaces in higher order perturbative calculations in relativistic renormalizable quantum field theories.
\end{abstract}

\section{Introduction}

Precise theoretical predictions within the Standard Model of elementary particles are indispensable for the concise understanding of the fundamental parameters of this physical theory and the discovery of its potential extensions. At the experimental side highly precise measurements exist at $e^{+} e^{-}$, $e p$ and $p p$-colliders as at LEP, HERA, and the LHC. In the near future the high luminosity phase of the LHC will even provide much more precise data. Other facilities, like the ILC [1] and a possible FCC [2], are currently planned. During the last three decades enormous efforts have been made to calculate key observables measured at these colliders at higher and higher accuracy, to meet the challenge provided by the accuracy of the experiments.

For zero-scale quantities currently analytic massless calculations can be performed at the five-loop and for massive calculations at the four-loop level. Single scale calculations are performed in both cases at the three-loop level. To perform these large scale calculations very demanding efforts are needed at the side of their automation, computer-algebraic implementation, and the use of highly efficient mathematical technologies. Therefore, the present problems can only be solved within a very close interdisciplinary cooperation

Deutsches Elektronen-Synchrotron, DESY, Platanenallee 6, D-15738 Zeuthen, Germany, e-mail: Johannes.Bluemlein@desy.de 
between experts in all these different fields and it cannot be the sole tasks for theoretical physicists anymore.

While at one-loop order the mathematical solution for many scattering processes has been known early, cf. 3/5, systematic representations at higher loop order turned out to be more difficult. The core problem concerns the analytic integration of Feynman parameter integrals. Here integration is understood as anti-differentiation. An essential question is to determine the final solution space to which the respective integrals do belong and its mathematical structure, and to find the irreducible objects through which the corresponding integrals are represented. Furthermore, one needs efficient mathematical and computer-algebraic technologies to map the given Feynman parameter integrals into the latter quantities.

In this paper we give a survey on the main technological steps to calculate higher loop zero- and single-scale quantities in renormalizable quantum field theories, with the focus on analytic integration techniques and the occurring function spaces. The systematic theory of integration in this field is vastly developing and many more new structures are expected to be revealed in the future at higher loop levels and by considering the production of more particles in the final state of the respective scattering processes. These calculations are needed to obtain stable theoretical predictions for the experimental precision measurements at the present and future colliders, which operate at high luminosity.

The paper is organized as follows. In Section 2 we summarize the main steps in multi-loop perturbative calculations. Different methods used in symbolic calculations of zero- and single-scale Feynman parameter integrals are described in Section 3 . In Section 4 a hierarchy of function spaces, mainly for single-scale integrals, is discussed which emerge in present multi-loop calculations. Here we consider as well the representations in Mellin- $N$ and $x$-space. Section 5 contains the conclusions 1

\section{Main Steps in Multi-Loop Perturbative Calculations}

In most of the large projects, which are currently dealt with, the Feynman diagrams are generated using packages like QGRAF [12] and performing the color algebra for the gauge groups using Color [13. Standardized algorithms to obtain Feynman parameterizations exist, cf. e.g. 14 16. At growing complexity, to perform the Dirac- and spin-algebra will be a challenge even to FORM [17 20]. One further maps the set of the contributing Feynman integrals to master integrals using the integration-by-parts (IBP) technique 21. based on Laporta's algorithm 22, of which several implementations exist, cf. e.g. [23 27] and others. The remaining main step is then the integration

1 For other recent surveys on integration methods for Feynman integrals see [6]11]. 
of the master integrals. One possibility to inspect the problem on hand, is to analyze the associated system of first order differential equations for the master integrals. Sometimes it is also useful to consider, in addition, the related system of linear difference equations. One may decouple these systems using the algorithms implemented in the packages [28, 29, as e.g. Zürcher's algorithm [30]. This leads to a single differential equation or difference equation of large order and degree and associated determining equations for the remaining master integrals. If the former equations can be factored at first order, it is known that the master integrals can be obtained in terms of indefinitely nested sums or iterated integrals over certain alphabets, which are revealed in the solution process, e.g. using difference field and ring theory [31 43], algorithmically implemented in the package Sigma [44,45]. This applies to a wide class of physical cases. Most of the integration and summation methods described in Section 3 apply to them and allow to obtain the integrals analytically in terms of the mathematical functions described in Section 4. Finally, efficient numerical representations of these functions have to be provided to obtain numerical predictions of the different observables for the experiments.

\section{Symbolic Integration of Feynman Parameter Integrals}

In the following we summarize main aspects of the analytic integration of multi-loop Feynman parameter integrals. Of course these integrals can also be evaluated numerically, without observing their particular analytic structure, to some accuracy and methods exist to separate the different pole contributions in $\varepsilon$, cf. e.g. [46 56], which we will not discuss in the following. These methods play a role, however, also for testing analytic results. In calculating all the integrals required to solve a large scale problem, it is usually necessary to combine different analytic methods, at least for the sake of efficiency. This requirement finally led to the creation of these methods. In the future even more and further refined technologies will be needed to solve more enhanced problems. Finally, one ends up with sets of irreducible functions which span the solutions, see Section 4. The numerical representation of these functions is necessary and will be discussed in Section 4.3 .

Non of the different techniques described in the following are of universal character. In particular the solution of the most advanced problems will need a combined and sensible use of various of them. All of them have to be handled with care to achieve a steady stepwise reduction of the problem on hand and to avoid to enlarge the complexity, given the limited time and memory resources for the corresponding computer algebraic calculations. This will also apply to future developments, since more complex calculations will require further new and advanced technologies.

Many of the formalisms described below lead to summation problems. Their solution requires dedicated and efficient algorithms in difference field 
theory as implemented in the packages Sigma 44,45, EvaluateMultiSums and SumProduction [57 59, see also 60].

\subsection{The PSLQ Method}

The PSLQ method applies to the solution of zero dimensional quantities, i.e. physical quantities given by pure numbers. If the pool of constants is known or can be guessed over which the corresponding quantity has a polynomial representation over $\mathbb{Q}$, a highly precise numerical representation of the quantity and the individual monomials allows to determine the corresponding rational coefficients, cf. 61. This method has been applied recently in a massive calculation of the five-loop QCD $\beta$-function [62. Here the individual master integrals certainly contain also constants of elliptic nature and probably beyond. However, they all cancel in the final result, which is spanned by multiple zeta values (MZVs) 63,64, more precisely by $\left\{\zeta_{2}, \zeta_{3}, \zeta_{5}, \zeta_{7}\right\}$, beyond pure rational terms. Let us illustrate the method by an example. We would like to determine the harmonic polylogarithm $H_{-1,0,0,1}(1)$, cf. Section 4.2. which is given by a polynomial of MZVs up to weight $w=4$. I.e. we have to apply the PSLQ method over all monomials up to $w=4$

$$
\left\{\ln (2), \zeta_{2}, \zeta_{3}, \operatorname{Li}_{4}\left(\frac{1}{2}\right)\right\} \text {. }
$$

An approximate numerical value of $H_{-1,0,0,1}(1)$ is

0.3395454690873598695906678484608602061387815339795751791304750

222490137419723806082682624196443182167020255697096551752247012

11749559277

and PSLQ yields

$$
H_{-1,0,0,1}(1)=-\frac{1}{12} \ln ^{4}(2)+\frac{1}{2} \ln ^{2}(2) \zeta_{2}+\frac{3}{5} \zeta_{2}^{2}-\frac{3}{4} \ln (2) \zeta_{3}-2 \operatorname{Li}_{4}\left(\frac{1}{2}\right) .
$$

In particular, monomials like $\ln (2), \ln ^{2}(2), \ln ^{3}(2), \zeta_{2}, \zeta_{3}$ do not contribute here. 


\subsection{Hypergeometric Functions and their Generalizations}

Simpler Feynman-parameter integrals have representations in terms of generalized hypergeometric functions 65 67] and their generalizations such as Appell-, Kampe-De-Feriet- and related functions 68 79. This is due to the hyperexponential nature of the Feynman-parameter integrals, implying real exponents due to the dimensional parameter $\varepsilon$. These representations map multiple integrals to single series (for generalized hypergeometric functions) and double infinite series (e.g. for Appell series), which finally have to be solved by applying summation theory. The simplest function is Euler's Betafunction implying the series of ${ }_{p+1} F_{p}$ functions

$$
\begin{aligned}
B\left(a_{1}, a_{2}\right)= & \int_{0}^{1} d t t^{a_{1}-1}(1-t)^{a_{2}-1} \\
{ }_{3} F_{2}\left(a_{1}, a_{2}, a_{3} ; b_{1}, b_{2} ; x\right)= & \frac{\Gamma\left(b_{2}\right)}{\Gamma\left(a_{3}\right) \Gamma\left(b_{2}-a_{3}\right)} \int_{0}^{1} d t t^{a_{3}-1}(1-t)^{-a_{3}+b_{2}-1} \\
& \times{ }_{2} F_{1}\left(a_{1}, a_{2} ; b_{1} ; t x\right)
\end{aligned}
$$

Representations of this kind are usually sufficient for massless and massive single-scale two-loop problems 80 83. In the case of three-loop ladder graphs Appell-functions are appearing [84,85]. There are some more classes of higher transcendental functions of this kind, which have been studied in the mathematical literature [71,72, 76]. The corresponding representations allow the expansion in the dimensional parameter $\varepsilon$. At a given level in the calculation of Feynman diagrams one will not find corresponding known function representations and one has to invoke other methods of integration. One way to derive analytic infinite sum representations are Mellin-Barnes integrals to which we turn now.

\subsection{Analytic Solutions using Mellin-Barnes Integrals}

The higher transcendental functions discussed in Section 3.2 have representations in terms of Pochhammer-Umlauf integrals [65, 86, 87] and related to it, by Mellin-Barnes integrals [88, 89. They are defined by

$$
\frac{1}{(a+b)^{\alpha}}=\frac{1}{\Gamma(\alpha)} \frac{1}{2 \pi i} \int_{-i \infty}^{i \infty} d z \Gamma(\alpha+z) \Gamma(-z) \frac{b^{z}}{a^{\alpha+z}}, \quad \alpha \in \mathbb{R}, \alpha>0,
$$

cf. e.g. 90. Here the contour integral is understood to be either being closed to the left or the right surrounding the corresponding singularities. The Mellin-Barnes decomposition is analogous to the binomial (series) expansion for $\alpha<0$. After its application, various more Feynman parameters can be 
integrated using the technique described in Section 3.2. In every application the decomposition introduces a number of infinite sums of depth one according to the residue theorem. There exist some packages for Mellin-Barnes integrals 91 94, allowing also for numerical checks. Finally all the produced sums have to be solved using multi-summation methods. Therefore one is advised to apply this method very carefully. Not all expressions generated by this method can be analytically summed using the presently know technologies, cf. [44, 45. Sometimes Mellin $-N$ space techniques may lead to elliptic structures, while $x$-space techniques do not, cf. [95, and sum-representations have to be cast back into definite integral representations first.

\subsection{Hyperlogarithms}

In a wide class of cases Feynman integrals can be represented by combinations of Kummer-Poincaré integrals 96-100 for (a part) of their expansion coefficients in $\varepsilon$. Let us assume one can isolate these terms, see 101, and forms a corresponding finite multi-integral. The method of hyperlogarithms 102 has originally intended to reorganize these integrals such that one can find a sequence of integrations being linear in the Feynman parameter on hand. If this is the case the result is given in terms of Kummer-Poincaré integrals. For a corresponding implementation see [103]. The method has first been applied to the usual massless Feynman integrals. A generalization for massive integrals also containing local operator insertions has been given in [104, with an implementation in [105. Here also certain non-linear Feynman parameter structures, breaking multi-linearity, could be integrated.

\subsection{The Method of Differential Equations}

In single-scale processes systems of ordinary differential equations for the master integrals are naturally obtained by the IBP-relations differentiating for a parameter $x{ }^{2}$ The master integrals may then be calculated by solving these systems under given physical boundary conditions, [106 109. One considers the system

$$
\frac{d}{d x}\left(\begin{array}{c}
f_{1} \\
\vdots \\
f_{n}
\end{array}\right)=\left(\begin{array}{ccc}
A_{11} & \ldots & A_{1, n} \\
\vdots & & \vdots \\
A_{n 1} & \ldots & A_{n, n}
\end{array}\right)\left(\begin{array}{c}
f_{1} \\
\vdots \\
f_{n}
\end{array}\right)+\left(\begin{array}{c}
g_{1} \\
\vdots \\
g_{n}
\end{array}\right),
$$

\footnotetext{
${ }^{2}$ Correspondingly, in the case of more parameters, partial differential equation systems are obtained.
} 
which may also be transformed into the scalar differential equation

$$
\sum_{k=0}^{n} p_{n-k}(x) \frac{d^{n-k}}{d x^{n-k}} f_{1}(x)=\bar{g}(x),
$$

with $p_{n} \neq 0$, and $(n-1)$ equations for the remaining solutions, which are fully determined by the solution $f_{1}(x)$. In setting up these systems one has to perform the expansion in $\varepsilon$ in parallel in the decoupling.

An important class of differential equations is formed by the first order factorizing systems, after applying the decoupling methods [8,30] encoded in Oresys 28, which appear as the simplest case. Eq. (7) may be transformed into Mellin space, decoupled there and solved using the efficient methods of the package Sigma, cf. Ref. [85].

The decoupled differential operator of (8) can be written in form of a combination of iterative integrals, cf. Section 4.2 ,

$$
\begin{aligned}
f_{1}(x)= & \sum_{k=1}^{n+1} \gamma_{k} g_{k}(x), \gamma_{k} \in \mathbb{C} \\
g_{k}(x)= & h_{0}(x) \int_{0}^{x} d y_{1} h_{1}\left(y_{1}\right) \int_{0}^{y_{1}} d y_{2} h_{2}\left(y_{2}\right) \ldots \int_{0}^{y_{k-2}} d y_{k-1} h_{k-1}\left(y_{k-1}\right) \\
& \times \int_{0}^{y_{k-1}} d y_{k} q_{k}\left(y_{k}\right)
\end{aligned}
$$

with $q_{k}(x)=0$ for $1 \leq k \leq m$. Further, $\gamma_{m+1}=0$ if $\bar{g}(x)=0$ in (8), and $\gamma_{m+1}=1$ and $q_{m+1}(x)$ being a mild variation of $\bar{g}(x)$ if $\bar{g}(x) \neq 0$. These solutions are d'Alembertian [110] since the master integrals appearing in quantum field theories obey differential equations with rational coefficients, the letters $h_{i}$, which constitute the iterative integrals, have to be algebraic. The solution can be computed using the package HarmonicSums [111. More generally, also Liouvillian solutions [112] can be calculated with HarmonicSums utilizing Kovacic's algorithm [113]. This algorithm has been applied in many massive three-loop calculations so far, see also [85, 114, 116.

If being transformed to the associated system of difference equations, the same holds, if this system is also first order factorizing. The solution of the remaining equations are directly obtained by the first solution.

In the multi-variate case, the $\varepsilon$-representation of a linear system of partial differential equations

$$
\partial_{m} f\left(\varepsilon, x_{n}\right)=A_{m}\left(\varepsilon, x_{n}\right) f\left(\varepsilon, x_{n}\right)
$$

is important, as has been recognized in Refs. [117, 118, see also [119]. The matrices $A_{n}$ can now be transformed in the non-Abelian case by

$$
A_{m}^{\prime}=B^{-1} A_{m} B-B^{-1}\left(\partial_{m} B\right),
$$


see also [120,121, and one now intends to find a matrix $B$ to transform (11) into the form

$$
\partial_{m} f\left(\varepsilon, x_{n}\right)=\varepsilon A_{m}\left(x_{n}\right) f\left(\varepsilon, x_{n}\right),
$$

if possible. This then allows solutions in terms of iterative integrals. A formalism for the basis change to the $\varepsilon$-basis has been proposed in 122 and implemented in the single-variate case in [123,124 and in the multi-variate case in [125.

\subsection{The Method of Arbitrary Large Moments}

In the case of single-scale problems the corresponding class of Feynman integrals depends on a real parameter $x \in[0,1]$, which is given e.g. as the ratio of two Lorentz invariants. For any power in $\varepsilon$ one would like to find the corresponding function in $x$ analytically. In a series of cases, cf. e.g. [116] 126 128, one may represent the solution in terms of a formal Taylor series in the variable $x$. The differential equations implied by the integration-by-parts method [21, 22, 25] 27] can now be turned into recurrences using the Taylor series ansatz. In solving the corresponding system one may generate a large number of Mellin moments for the different projections on the individual color factors and multiple zeta values 64. This is the case independently of the fact that the corresponding $x-$ or $N$-space solution is given by iterative integrals or iterative-noniterative integrals. The corresponding method has been described in Ref. [129]. These moments can then be used as an input to the method described in Section 3.7 to find the associated difference equations. In some applications for single scale massive three-loop integrals 114 8000 moments could be calculated. This is by far more than possible using standard methods like Mincer [130, MATAD [131] or Q2E [132, 133]. Based on this number of moments, the formal power series may be used as highly precise semi-analytic numeric representations, in case the corresponding series expansion has been performed for the physical quantity to be evaluated. If analytic continuations are still necessary, the method cannot be applied directly.

\subsection{Guessing One-dimensional Integrals}

As has been described in Section 3.6 single-variate multiple Feynman parameter integrals can be either expanded into formal Taylor series or can be Mellin-transformed 


$$
G(N)=\mathbf{M}[f(x)](N)=\int_{0}^{1} d x x^{N-1} f(x) .
$$

In both cases one tries now to find the associated difference equation 134 to the set of moments, e.g. $\{G(2), G(4), \ldots ., G(2 m)\}, m \in \mathbb{N}[135] 138$. Indeed such an equation exists in many cases, as e.g. for (massive) operator matrix elements [139], but also for single-scale Wilson coefficients, Ref. [140. If a suitably large number of moments has been calculated analytically the associated series of rational numbers can now be used as input for the guessing algorithm [141, which is also available in Sage [142, exploiting the fast integer algorithms available there. The method finally returns the wanted difference equation, and tests it by a larger series of further moments. This method has been applied in Ref. [143] to obtain from more than 5000 moments the massless unpolarized three-loop anomalous dimensions and Wilson coefficients in deep-inelastic scattering [140, 144, 145]. Recently, the method has been applied $a b$ initio in the calculation of three-loop splitting functions 146 . and the massive two- and three-loop form factor [116, 147. In the case of a massive operator matrix element 8000 moments [114] could be calculated and difference equations were derived for all contributing color and $\zeta$-value structures. Analytic solutions can be found using the package Sigma [4, 45, provided the problem is solvable in difference field theory. In other cases at least the first order factorizing parts can be factored off. Other techniques are then needed to determine the remainder part of the solution.

\subsection{The Almkvist-Zeilberger Algorithm}

Since Feynman parameter integrals, depending on an additional parameter $x$, can be given as integrals over $\left\{\left.x_{i}\right|_{1=1} ^{n}\right\} \in[0,1]^{n}$, they form the multiintegral $I(x)$, depending also on $\varepsilon$. The dependence on the real parameter $x$ may be transformed into one on an integer parameter $N$, see Section 3.6. The Almkvist-Zeilberger algorithm [148, 149] is providing a method to find either an associated differential equation for $I(x)$ or a difference equation for $I(N)$, the coefficients of which are either polynomials in $\{x, \varepsilon\}$ or $\{N, \varepsilon\}$,

$$
\begin{aligned}
\sum_{l=0}^{m} P_{l}(x, \varepsilon) \frac{d^{l}}{d x^{l}} I(x, \varepsilon) & =N(x, \varepsilon) \\
\sum_{l=0}^{m} R_{l}(N, \varepsilon) I(N+l, \varepsilon) & =M(N, \varepsilon) .
\end{aligned}
$$

Both equations may be inhomogeneous, where the inhomogeneities emerge as known functions from lower order problems. An optimized and improved 
algorithm for the input class of Feynman integrals has been implemented in the MultiIntegrate package 85, 150. It can either produce homogeneous equations of the form (15/16) or equations with an inhomogeneity formed out of already known functions.

\subsection{Iterative-Noniterative Integrals and Elliptic Solutions}

Non-first order factorizing systems of differential or difference equations for the master integrals, cf. Section 3.5 occur at a certain order in massive Feynman diagram calculations. Well-known examples for this are the sun-rise integral, cf. e.g. [151-157, the kite integral [158 160], the three-loop QCDcorrections to the $\rho$-parameter 161 163, and the three-loop QCD corrections to the massive operator matrix element $A_{Q g}$ [114]. After separating the first-order factorizing factors a Heun differential equation [164 remains in the case of the $\rho$-parameter. One may write the corresponding solution also using ${ }_{2} F_{1}$-functions with rational argument [161,165] and rational parameters. It is now interesting to see whether these solutions can be expressed in terms of complete elliptic integrals, which can be checked algorithmically using the triangle group [166.

In the examples mentioned one can find representations in terms of complete elliptic integrals of the first and second kind, $\mathbf{K}$ and $\mathbf{E}$, cf. [167, 168, and the question arises whether an argument translation allows for a representation through only $\mathbf{K}$. Criteria for this have been given in [169, 170]. In the case of the three-loop QCD-corrections to the $\rho$-parameter, however, this is not possible.

The homogeneous solution of the Heun equations are given by ${ }_{2} F_{1}-$ solutions $\psi_{k}^{(0)}(x), k=1,2$, at a specific rational argument. These integrals cannot be represented such that the variable $x$ just appears in the boundaries of the integral. The inhomogeneous solution reads

$$
\psi(x)=\psi_{1}^{(0)}(x)\left[C_{1}-\int d x \psi_{2}^{(0)}(x) \frac{N(x)}{W(x)}\right]+\{1 \rightarrow 2\},
$$

with $N(x)$ and $W(x)$ the inhomogeneity and the Wronskian. $C_{1,2}$ are the integration constants. Through partial integration the ratio $N(x) / W(x)$ can be transformed into an iterative integral. Since $\psi_{k}^{(0)}(x)$ cannot be written as iterative integrals, $\psi(x)$ is obtained as an iterative non-iterative integral [161,171] of the type

$$
\begin{aligned}
& \mathbb{H}_{a_{1}, \ldots, a_{m-1} ; a_{m}, F_{m}\left(r\left(y_{m}\right)\right), a_{m+1}, \ldots a_{q}}(x)= \\
& \int_{0}^{x} d y_{1} f_{a_{1}}\left(y_{1}\right) \int_{0}^{y_{1}} d y_{2} \ldots \int_{0}^{y_{m-1}} d y_{m} f_{a_{m}}\left(y_{m}\right) F_{m}\left[r\left(y_{m}\right)\right] H_{a_{m+1}, \ldots, a_{q}}\left(y_{m}\right),(18)
\end{aligned}
$$


with $r(x)$ a rational function and $F_{m}$ a non-iterative integral. Usually more than one non-iterative integral will appear in (18). $F_{m}$ denotes any non-iterative integral, implying a very general representation, cf. [161. $3^{3}$ In Ref. 173, an $\varepsilon$-form for the Feynman diagrams of elliptic cases has been found recently. However, transcendental letters contribute here. This is in accordance with our earlier finding, Eq. (18), which, as well is an iterative integral over all objects between the individual iterations and to which now also the non-iterative higher transcendental functions $F_{m}\left[r\left(y_{m}\right)\right]$ contribute. One may obtain fast convergent representations of $\mathbb{H}(x)$ by overlapping series expansions around $x=x_{0}$ outside possible singularities, see Ref. [161] for details.

Let us return to the elliptic case now. Here one one may transform the kinematic variable $x$ occurring as $\mathbf{K}\left(k^{2}\right)=\mathbf{K}(r(x))$ into the variable $q=$ $\exp [i \pi \tau]$ analytically with

$$
k^{2}=r(x)=\frac{\vartheta_{2}^{4}(q)}{\vartheta_{3}^{4}(q)},
$$

by applying a 3rd order Legendre-Jacobi transformation, where $\vartheta_{l}, l=1, \ldots, 4$ denote Jacobi's $\vartheta$-functions and $\operatorname{Im}(\tau)>0$. In this way Eq. (17) is rewritten in terms of the new variable. The integrands are given by products of meromorphic modular forms, cf. [174 176], which can be written as a linear combination of ratios of Dedekind's $\eta$-function

$$
\eta(\tau)=q^{\frac{1}{12}} \prod_{k=1}^{\infty}\left(1-q^{2 k}\right) .
$$

Depending on the largest multiplier $k \in \mathbb{N}, k_{m}$, of $\tau$ in the argument of the $\eta$-function, the solution transforms under the congruence subgroup $\Gamma_{0}\left(k_{m}\right)$. One can perform Fourier expansions in $q$ around the different cusps of the problem, cf. [177, 178].

In the case that the occurring modular forms are holomorphic, one obtains representations in Eisenstein series with character, while in the meromorphic case additional $\eta$-factors in the denominators are present. In the former case the $q$-integrands can be written in terms of elliptic polylogarithms in the representation [155, 156.

$$
\operatorname{ELi}_{n, m}(x, y)=\sum_{k=1}^{\infty} \sum_{l=1}^{\infty} \frac{x^{k}}{k^{n}} \frac{y^{l}}{l^{m}} q^{k l}
$$

and products thereof, cf. [156. The corresponding $q$-integrals can be directly performed. The solution (17) usually appears for single master integrals. Other master integrals are obtained integrating further other letters, so that finally representations by $\mathbb{H}(x)$ occur. Iterated modular forms, resp. Eisen-

\footnotetext{
3 This representation has been used in a more special form also in 172 later.
} 
stein series, have been also discussed recently in [179,180]. Efficient numerical calculations of modular forms based on $q$-series were obtained in [181.

For systems which factorize only to 3rd and higher order much less is known.

\subsection{Iterative Integrals of Functions with More Variables}

The occurrence of several masses or additional external non-factorizing scales in higher order loop- and phase-space integrals leads in general to rational and root-valued letters with real parameter letters in the contributing alphabet, cf. [95, 182, 185. In the case of the loop integrals one obtains letters of the kind

$$
\frac{1}{1-x(1-\eta)}, \frac{\sqrt{x(1-x)}}{\eta+x(1-\eta)}, \sqrt{x(1-\eta(1-x)}, \quad \eta \in[0,1] .
$$

The iterative integrals and constants which appeared in 95, 182 could finally be all integrated to harmonic polylogarithms containing complicated arguments, at least up to one remaining integration, which allows their straightforward numerical evaluation.

In the case of phase space integrals with more scales, e.g. 183, 184, also letters contribute, which may imply incomplete elliptic integrals and iterated structures thereof. Contrary to the functions obtained in Section 3.9 these are still iterative integrals, because the boundaries of the phase-space integrals are real parameters and not constants. The integrands could not by rationalized completely by variable transformations, see also [186]. Contributing letters are e.g.

$$
\frac{x}{\sqrt{1-x^{2}} \sqrt{1-k^{2} x^{2}}}, \frac{x}{\sqrt{1-x^{2}} \sqrt{1-k^{2} x^{2}}\left(k^{2}\left(1-x^{2}\left(1-z^{2}\right)\right)-z^{2}\right)},
$$

with $k, z \in[0,1]$. The corresponding iterative integrals are called Kummerelliptic integrals. They are derived using the techniques described in Refs. 187 189 .

\section{A Series of Function Spaces}

Intermediary and final results for zero- and single-scale multi-loop calculations have representations by special functions as polynomials over $\mathbb{Q}$. In the case of of zero-scale quantities these are special numbers. For single scale quantities one either uses finite nested sum representations in Mellin $N$-space or iterative integral representations in $x$-space. Here $x$ denotes a Lorentz in- 
variant ratio of two physical quantities. Both spaces are related to each other by the Mellin transform (14), where $f(x)$ denotes an iterative integral. The zero-scale quantities can be obtained e.g. in the limit $N \rightarrow \infty$ of these Mellin transforms or by the values $f(x=1)$.

\subsection{Classes of Nested Sums}

The methods described in Section 3 very often lead to finite nested sum representations for which algorithms exist [44, 45] to cast these sums into indefinitely nested sums. They are given by

$$
S_{b, \mathbf{a}}(N)=\sum_{k=1}^{N} g_{b}(k) S_{\mathbf{a}}(n), \quad S_{\emptyset}=1, g_{c} \in \overline{\mathfrak{A}},
$$

with $\overline{\mathfrak{A}}$ the associated alphabet of functions. The sums obey quasi-shuffle relations 190, 191. The simplest structures are the finite harmonic sums 192, 193, where $g_{b}(k)=(\operatorname{sign}(b))^{k} / k^{|b|}, \quad b \in \mathbb{N} \backslash\{0\}$. A generalization is obtained in the cyclotomic case [194. Here the characteristic summands are $g_{a, b, c}(k)=( \pm 1)^{k} /(a k+b)^{c}$, with $a, b, c \in \mathbb{N} \backslash\{0\}$. Further, the generalized harmonic sums have letters of the type $b^{k} / k^{c}$, with $c \in \mathbb{N} \backslash\{0\}, b \neq 0, b \in \mathbb{R}$, 195. Another generalization are nested finite binomial and inverse-binomial sums, containing also other sums discussed before. An example is given by

$$
\begin{aligned}
& \sum_{i=1}^{N}\left(\begin{array}{c}
2 i \\
i
\end{array}\right)(-2)^{i} \sum_{j=1}^{i} \frac{1}{j\left(\begin{array}{c}
2 j \\
j
\end{array}\right)} S_{1,2}\left(\frac{1}{2}, 1\right)(j)=\int_{0}^{1} d x \frac{(-x)^{N}-1}{x+1} \sqrt{\frac{x}{8-x}} \\
& \times\left[\mathrm{H}_{\mathrm{w}_{12}, 1,0}(x)-2 \mathrm{H}_{\mathrm{w}_{13}, 1,0}(x)-\zeta_{2}\left(\mathrm{H}_{\mathrm{w}_{12}}(x)-2 \mathrm{H}_{\mathrm{w}_{13}}(x)\right)\right] \\
& -\frac{5 \zeta_{3}}{8 \sqrt{3}} \int_{0}^{1} d x \frac{(-2 x)^{N}-1}{x+\frac{1}{2}} \sqrt{\frac{x}{4-x}}+c_{1} \int_{0}^{1} d x \frac{(-8 x)^{N}-1}{x+\frac{1}{8}} \sqrt{\frac{x}{1-x}},
\end{aligned}
$$

with $c_{1} \approx 0.10184720 \ldots$, cf. [189]. Here the indices $\mathrm{w}_{\mathrm{k}}$ label specific letters given in 189. Infinite binomial and inverse binomial sums have been considered in 196, 197. Given the general structure of (24) many more iterated sums can be envisaged and may still appear in even higher order calculations.

\subsection{Classes of Iterated Integrals}

Iterated integrals have the structure 


$$
\mathrm{H}_{b, \mathbf{a}}(x)=\int_{0}^{x} d y f_{b}(y) \mathrm{H}_{\mathbf{a}}(y), \quad \mathrm{H}_{\emptyset}=1, f_{c} \in \mathfrak{A},
$$

where $f_{c}$ are real functions and are the letters of the alphabet $\mathfrak{A}$. Iterated integrals obey shuffle relations [191,198, which allows to represent them over a multinomial basis of fewer terms.

The simplest iterative integral having been considered in quantum field theory are the Nielsen integrals for the two-letter alphabets $\{1 / x, 1 /(1-x)\}$ or $\{1 / x, 1 /(1+x)\}$ 199 202, covering also the polylogarithms 202 204. This class has later been extended to the harmonic polylogarithms [205] build over the alphabet $\{1 / x, 1 /(1-x), 1 /(1+x)\}$. A further extension is to the real representations of the cyclotomic polylogarithms, with $\left\{1 / x, 1 / \Phi_{k}(x)\right\}$ [194, where $\Phi_{k}(x)$ denotes the $k$ th cyclotomic polynomial. Another extension is given by Kummer-Poincaré iterative integrals over the alphabet $\{1 /(x-$ $\left.\left.a_{i}\right), \quad a_{i} \in \mathbb{C}\right\},[96-100$. Properties of these functions have been studied in Refs. 195, 206]. In general one may have also more general denominator polynomials $P(x)$, which one can factor into

$$
P(x)=\prod_{k=1}^{n}\left(x-a_{k}\right) \prod_{l=1}^{m}\left(x^{2}+b_{l} x+c_{l}\right), \quad a_{k}, b_{l}, c_{l} \in \mathbb{R}
$$

in real representations. One then performs partial fractioning for $1 / P(x)$ and forms iterative integrals out of the obtained letters. Further classes are found for square-root valued letters as studied e.g. in Ref. [189. In multi-scale problems, cf. e.g. 95, 183 185] and Section 3.10, further root-valued letters appear, like also the Kummer-elliptic integrals [184].

\subsection{Classes of Associated Special Numbers}

For the sums of Section 4.1 which are convergent in the limit $N \rightarrow \infty$ and the iterated integrals of Section 4.2 which can be evaluated at $x=1$ one obtains two sets of special numbers. They span the solution spaces for zeroscale quantities and appear as boundary values for single-scale problems. Examples for these special numbers are the multiple zeta values 64, associated to the harmonic sums and harmonic polylogarithms, special generalized numbers 195. like $\mathrm{Li}_{2}(1 / 3)$, associated to generalized sums and to KummerPoincaré iterated integrals, special cyclotomic numbers 194] like Catalan's number, special binomial numbers [189, as e.g. $\operatorname{arccot}(\sqrt{7})$, and special constants in the elliptic case [161,207. The latter numbers are given by integrals involving complete elliptic integrals at special rational arguments and related functions. In general these numbers obey more relations than the finite sums and iterated integrals. One may use the PSLQ-method to get a first infor- 
mation on relations between these numbers occurring in a given problem and proof the conjectured relations afterwards.

\subsection{Numerical Representations}

Physical observables based on single scale quantities can either be represented in Mellin $N$-space or $x$-space. Representations in Mellin $N$-space allow the exact analytic solution of evolution equations 208] and schemeinvariant evolution equations can be derived in this way [209, 210]. The $x^{-}$ space representation is then obtained by a single numerical integral around the singularities of the respective quantity for $N \in \mathbb{C}$, cf. [208, requiring to know the complex representation of the integrand in $N$-space. In the case of harmonic sums semi-numerical representations were given in [211, 212]. Furthermore, it is known that basic harmonic sums, except of $S_{1}(N)$, which is represented by the Digamma function, and its polynomials, have a representation by factorial series [213, 214, which has been used in [215, 216] for their asymptotic representation, see also 217. One uses then the recursion relations, which can be obtained from (24), to move $N \in \mathbb{C}$ from the asymptotic region to the desired point on the integration contour in the analyticity region of the problem. This can be done for the sums of the type being described in Refs. 189, 194, 195] as well, since also in this case asymptotic expansions can be provided, at least for certain combinations of sums occurring in the respective physical problem, cf. [85, 104. In the case that the corresponding relations are not given in tabulated form, they can be calculated using the package HarmonicSums [111, 150, 189, 192, 195, 218, 219. Relations for harmonic sums are also implemented in summer [192, and for generalized harmonic sums in nestedsums [220, Xsummer [221], and PolyLogTools 222].

In other applications one may want to work in $x$-space directly. Here numerical representations are available for the Nielsen integrals 200, the harmonic polylogarithms [223 227, the Kummer-Poincaré iterative integrals [227, and the cyclotomic harmonic polylogarithms [116. These representations are also useful to lower the number of numerical integrations for more general problems, e.g. in the multi-variate case. The relations for the corresponding quantities are implemented for the harmonic polylogarithms in [205, 224] and for all iterative integrals mentioned, including general iterative integrals, in the package HarmonicSums.

\section{Conclusions}

In parallel to the analytic higher-loop calculations in Quantum Field Theory the associated mathematical methods have been developed by theoretical 
physicists and mathematicians since the 1950ies. We witness a very fast development since the late 1990ies approaching difficult massive problems at two-loop and higher order and massless problems form three loops onward. The classical methods of polylogarithms and Nielsen-integrals which were standard means, turned out to be not sufficient anymore. Since then more and more special number- and function spaces have been revealed, studied and were brought to flexible practical use in very many applications. Moreover, a wide host of analytic integration and summation methods has been developed during a very short period. In this way very large physics problems could be solved analytically - a triumph of the exact sciences, also thanks to various groundbreaking methods in computer algebra. In this context the goal is to improve the accuracy of the fundamental parameters of the Standard Model of the elementary particles further. Within the present projects this concerns in particular the relative precision of the strong coupling constant $\alpha_{s}\left(M_{Z}^{2}\right)$ to less than $1 \%$ and of the $\overline{\mathrm{MS}}$ mass of the charm quark to better than $1.5 \%$.

At even higher loop order and for more separated final state legs, introducing more masses and kinematic invariants, one expects further mathematical structures to contribute. Possible structures of this kind could be Abel-integrals [228] and integrals related to K3-surfaces [229. More inclusive methods, like the method of differential equations, can certainly determine the degree of non-factorization of a physical problem. However, one would like to know in a closer sense the respective analytic solution. Here cutting methods can be of use since the underlying integrands can be systematically related to the final integral by (various) Hilbert-transform [230 232] 4 In this way integrand structures are revealed, which are somewhat hidden in the case of differential equations. This method has been advocated early by M. Veltman 234, see also 235.

This process to master highly complex Feynman integrals using analytic methods is of course just at the beginning and will develop further given the present and future challenges in the field. All of these results put experimental analyses in precision measurements at the high energy colliders into the position to analyze the data with much reduced theory errors and we will get far closer in our insight into the structure of the micro cosmos to reveal its ultimate laws. The interdisciplinary joined effort by mathematicians, theoretical and experimental particle physicists and experts in computer algebra makes this possible and allows to answer quite a series of fundamental scientific questions of our time.

I would like to give my warmest thanks to Peter Paule for his continuous collaboration and support to the DESY-RISC collaboration, starting with our first contacts in 2005, arranged by Bruno Buchberger. This scientific symbiosis has produced a large number of methods to tackle quite a series of difficult problems since, and is continuing to do so in the future. Physics,

\footnotetext{
${ }^{4}$ For a recent application to the one-loop case, see e.g. [233].
} 
mathematics, and computer algebra profit from this and reach new horizons, which, not at all, could have been imagined. In this way we follow together the motto D. Hilbert has given to us:

Wir müssen wissen. Wir werden wissen.

Le but unique de la science, c'est l'honneuer de l'esprit humain 5

Acknowledgment. I would like to thank J. Ablinger, D. Broadhurst, A. De Freitas, D. Kreimer, A. von Manteuffel, P. Marquard, S.-O. Moch, W.L. van Neerven, P. Paule, C. Schneider, K. Schönwald, J. Vermaseren and S. Weinzierl for countless fruitful discussions. This work was supported by the EU TMR network SAGEX Marie Skłodowska-Curie grant agreement No. 764850 and COST action CA16201: Unraveling new physics at the LHC through the precision frontier.

\section{References}

1. The ILC: https://en.wikipedia.org/wiki/International_Linear_Collider J.A. Aguilar-Saavedra et al. [ECFA/DESY LC Physics Working Group], TESLA: The Superconducting electron positron linear collider with an integrated x-ray laser laboratory. Technical design report. Part 3. Physics at an $e^{+} e^{-}$linear collider, hep-ph/0106315;

E. Accomando et al. [ECFA/DESY LC Physics Working Group], Phys. Rept. 299 (1998) 1-78 [hep-ph/9705442].

2. The Future Circular Collider, https://en.wikipedia.org/wikiFuture_Circular_Collider;

TH FCC-ee design study, http://tlep.web.cern.ch.

3. G. 't Hooft and M.J.G. Veltman, Nucl. Phys. B153 (1979) 365-401.

4. G. 't Hooft and M.J.G. Veltman, NATO Sci. Ser. B 4 (1974) 177-322.

5. M.J.G. Veltman, Diagrammatica: The Path to Feynman rules, Cambridge Lect. Notes Phys. 4, (Cambridge University Press, Cambridge, 1994).

6. S. Weinzierl, Introduction to Feynman Integrals, arXiv:1005.1855 [hep-ph].

7. J. Ablinger, J. Blümlein, and C. Schneider, J. Phys. Conf. Ser. 523 (2014) 012060, [arXiv: 1310.5645 [math-ph]].

8. J. Ablinger and J. Blümlein, in: Computer Algebra in Quantum Field Theory. Integration, Summation and Special Functions, (Springer, Wien, 2012), 1-32, Eds. C. Schneider and J. Blümlein, [arXiv: 1304.7071 [math-ph]].

9. S. Weinzierl, in: Computer Algebra in Quantum Field Theory. Integration, Summation and Special Functions, (Springer, Wien, 2012), 381-406, Eds. C. Schneider and J. Blümlein, [arXiv:1301.6918 [hep-ph]].

10. C. Duhr, in: Journeys Through the Precision Frontier: Amplitudes for Colliders, 2014 TASI Lectures, eds. L. Dixon and F. Petriello, 419-476 (World Scientific, Singapore, 2015) [arXiv:1411.7538 [hep-ph]].

11. J. Blümlein and C. Schneider, Int. J. Mod. Phys. A 33 (2018) no.17, 1830015 [arXiv:1809.02889 [hep-ph]].

12. P. Nogueira, J. Comput. Phys. 105 (1993) 279-289.

5 Jacobi to Legendre, July 2nd, 1830. 
13. T. van Ritbergen, A. Schellekens, and J.A.M. Vermaseren, Int. J. Mod. Phys. A14 (1999) 41-96, [hep-ph/9802376].

14. N. Nakanishi, Graph Theory and Feynman Integrals, (Gordon and Breach, New York, 1971).

15. S. Lefschetz, Applications of Algebraic Topology: Graphs and Networks, the PicardLefschetz Theory an Feynman Integrals, (Springer, Berlin, 1975).

16. C. Bogner and S. Weinzierl, Int. J. Mod. Phys. A25 (2010) 2585-2618, [arXiv:1002.3458[hep-ph]]

17. J.A.M. Vermaseren, New features of FORM, math-ph/0010025.

18. M. Tentyukov, D. Fliegner, M. Frank, A. Onischenko, A. Retey, H.M. Staudenmaier and J.A.M. Vermaseren, AIP Conf. Proc. 583 (2002) no.1, 202 [cs/0407066 [cs-sc]].

19. M. Tentyukov and J.A.M. Vermaseren, Comput. Phys. Commun. 181 (2010) 1419-1427, [hep-ph/0702279]

20. B. Ruijl, U. Takahiro, and J.A.M. Vermaseren, FORM version 4.2, arXiv: 1707.06453[hep-ph].

21. K.G. Chetyrkin and F.V. Tkachov, Nucl. Phys. B192 (1981) 159-204.

22. S. Laporta, Int. J. Mod. Phys. A15 (2000) 5087-5159, [hep-ph/0102033]

23. A. Smirnov, JHEP 10 (2008) 107, [arXiv: 0807.3243[hep-ph]]

24. A.V. Smirnov and F.S. Chuharev, FIRE6: Feynman Integral REduction with Modular Arithmetic, arXiv:1901.07808 [hep-ph].

25. C. Studerus, Comput. Phys. Commun. 181 (2010) 1293-1300, [arXiv: 0912.2546 [physics.comp-ph]]

26. A. von Manteuffel and C. Studerus, Reduze 2 - Distributed Feynman Integral Reduction, arXiv:1201.4330 [hep-ph].

27. P. Marquard and D. Seidel, The Crusher algorithm, (unpublished).

28. S. Gerhold, Uncoupling systems of linear Ore operator equations, Master's thesis, RISC, J. Kepler University, Linz, 2002.

29. A. Bostan, F. Chyzak, É. de Panafieu, Complexity Estimates for Two Uncoupling Algorithms, Proceedings of ISSAC'13, Boston, June 2013.

30. B. Zürcher, Rationale Normalformen von pseudo-linearen Abbildungen, Master's thesis, Mathematik, ETH Zürich (1994).

31. M. Karr, J. ACM 28 (1981) 305-350.

32. M. Bronstein, J. Symbolic Comput. 29 (2000), no. 6 841-877.

33. C. Schneider, Symbolic Summation in Difference Fields, Ph.D. Thesis RISC, Johannes Kepler University, Linz technical report 01-17 (2001).

34. C. Schneider, An. Univ. Timisoara Ser. Mat.-Inform. 42 (2004) 163-179.

35. C. Schneider, J. Differ. Equations Appl. 11 (2005) 799-821.

36. C. Schneider, Appl. Algebra Engrg. Comm. Comput. 16 (2005) 1-32.

37. C. Schneider, J. Algebra Appl. 6 (2007) 415-441.

38. C. Schneider, Clay Math. Proc. 12 (2010) 285-308 [arXiv:0904.2323 [cs.SC]]. [arXiv:0904.2323].

39. C. Schneider, Ann. Comb. 14 (2010) 533-552, [arXiv:0808.2596].

40. C. Schneider, in: Computer Algebra and Polynomials, Applications of Algebra and Number Theory, J. Gutierrez, J. Schicho, M. Weimann (ed.), Lecture Notes in Computer Science (LNCS) 8942 (2015), 157-191, [arXiv:1307.7887 [cs.SC]].

41. C. Schneider, J. Symbolic Comput. 43 (2008) 611-644 [arXiv:0808.2543].

42. C. Schneider, J. Symb. Comput. 72 (2016) 82-127, [arXiv:1408.2776 [cs.SC]].

43. C. Schneider, J. Symb. Comput. 80 (2017) 616-664. [arXiv:1603.04285 [cs.SC]].

44. C. Schneider, Sém. Lothar. Combin. 56 (2007) 1-36, article B56b

45. C. Schneider, in: Computer Algebra in Quantum Field Theory: Integration, Summation and Special Functions, Texts and Monographs in Symbolic Computation eds. C. Schneider and J. Blümlein (Springer, Wien, 2013), 325-360 [arXiv:1304.4134 $[\mathrm{cs} . \mathrm{SC}]]$. 
46. T. Binoth and G. Heinrich, Nucl. Phys. B585 (2000) 741-759, [hep-ph/ 0004013].

47. Z. Nagy and D.E. Soper, Phys. Rev. D74 (2006) 093006, [hep-ph/0610028].

48. C. Anastasiou, S. Beerli, and A. Daleo, JHEP 05 (2007) 071, [hep-ph/0703282].

49. A.V. Smirnov and M.N. Tentyukov, Comput. Phys. Commun. 180 (2009) 735746, [arXiv: 0807.4129[hep-ph]].

50. J. Carter and G. Heinrich, Comput. Phys. Commun. 182 (2011) 1566-1581, [arXiv:1011.5493[hep-ph]]

51. A.V. Smirnov, V.A. Smirnov, and M. Tentyukov, Comput. Phys. Commun. 182 (2011) 790-803, [arXiv:0912.0158[hep-ph]].

52. S. Becker, C. Reuschle, and S. Weinzierl, JHEP 12 (2010) 013, [arXiv:1010.4187[hep-ph]].

53. S. Becker, C. Reuschle, and S. Weinzierl, JHEP 1207 (2012) 090, [arXiv:1205.2096[hep-ph]].

54. S. Becker, D. Götz, C. Reuschle, C. Schwan, and S. Weinzierl, Phys. Rev. Lett. 108 (2012) 032005, [arXiv:1111.1733[hel-ph]]

55. A.V. Smirnov, Comput. Phys. Commun. 204 (2016) 189-199, [arXiv:1511.03614[hep-ph]]

56. S. Borowka, T. Gehrmann and D. Hulme, JHEP 1808 (2018) 111 [arXiv:1804.06824 [hep-ph]].

57. J. Ablinger, J. Blümlein, S. Klein, and C. Schneider, Nucl. Phys. Proc. Suppl. 205-206 (2010) 110-115, [arXiv:1006.4797[math-ph]]

58. J. Blümlein, A. Hasselhuhn, and C. Schneider, PoS (RADCOR2011) 032, [arXiv: 1202.4303 [math-ph]]

59. C. Schneider, J. Phys. Conf. Ser. 523 (2014) 012037, [arXiv:1310.0160[cs.Sc]]

60. C. Schneider, Contribution to this volume.

61. H.R.P. Ferguson and D.H. Bailey, A Polynomial Time, Numerically Stable Integer Relation Algorithm, RNR Techn. Rept. RNR-91-032, Jul. 14, 1992.

62. T. Luthe, A. Maier, P. Marquard, and Y. Schröder, JHEP 10 (2017) 166, [arXiv: 1709.07718 [hep-ph]]

63. J.M. Borwein, D.M. Bradley, D.J. Broadhurst, and P. Lisonek, Trans. Am. Math. Soc. 353 (2001) 907-941, [math/9910045].

64. J. Blümlein, D. Broadhurst, and J.A.M. Vermaseren, Comput. Phys. Commun. 181 (2010) 582-625, [arXiv: 0907.2557 [math-ph]].

65. F. Klein, Vorlesungen über die hypergeometrische Funktion, Wintersemester 1893/94, Die Grundlehren der Mathematischen Wissenschaften 39, (Springer, Berlin, 1933).

66. W.N. Bailey, Generalized Hypergeometric Series, (Cambridge University Press, Cambridge, 1935)

67. L.J. Slater, Generalized hypergeometric functions, (Cambridge University Press, Cambridge, 1966).

68. P. Appell and J. Kampé de Fériet, Fonctions Hypergéométriques et Hypersphériques, Polynomes D' Hermite, (Gauthier-Villars, Paris, 1926).

69. P. Appell, Les Fonctions Hypergëométriques de Plusieur Variables, (GauthierVillars, Paris, 1925).

70. J. Kampé de Fériet, La fonction hypergëométrique, (Gauthier-Villars, Paris, 1937).

71. H. Exton, Multiple Hypergeometric Functions and Applications, (Ellis Horwood, Chichester, 1976).

72. H. Exton, Handbook of Hypergeometric Integrals, (Ellis Horwood, Chichester, 1978).

73. M.J. Schlosser, in: Computer Algebra in Quantum Field Theory: Integration, Summation and Special Functions, C. Schneider, J. Blümlein, Eds., p. 305-324, (Springer, Wien, 2013) [arXiv:1305.1966 [math.CA]]. 
74. C. Anastasiou, E.W.N. Glover, and C. Oleari, Nucl. Phys. B572 (2000) 307360, [hep-ph/9907494]

75. C. Anastasiou, E.W.N. Glover, and C. Oleari, Nucl. Phys. B565 (2000) 445467, [hep-ph/9907523]

76. H.M. Srivastava and P.W. Karlsson, Multiple Gaussian Hypergeometric Series, (Ellis Horwood, Chicester, 1985).

77. G. Lauricella, Rediconti del Circolo Matematico di Palermo, 7 (S1) (1893) 111158.

78. S. Saran, Ganita 5 (1954) 77-91.

79. S. Saran, Acta Math. 93 (1955) 293-312.

80. R. Hamberg, W.L. van Neerven, and T. Matsuura, Nucl. Phys. B359 (1991) 343-405, [Erratum: Nucl. Phys. B644 (2002) 403-404].

81. R. Hamberg, Second order gluonic contributions to physical quantities, Ph.D. Thesis, Leiden University, 1991.

82. M. Buza, Y. Matiounine, J. Smith, R. Migneron, and W.L. van Neerven, Nucl. Phys. B472 (1996) 611-658, [hep-ph/9601302]

83. I. Bierenbaum, J. Blümlein, and S. Klein, Nucl. Phys. B780 (2007) 40-75, $[$ hep-ph/0703285]

84. J. Ablinger, J. Blümlein, A. Hasselhuhn, S. Klein, C. Schneider, and F. Wißbrock, Nucl. Phys. B864 (2012) 52-84, [arXiv:1206.2252[hep-ph]],

85. J. Ablinger, A. Behring, J. Blümlein, A. De Freitas, A. von Manteuffel, and C. Schneider, Comput. Phys. Commun. 202 (2016) 33-112, [arXiv:1509.08324 [hep-ph]]

86. L. Pochhammer, Math. Ann. 35 (1890) 495-526.

87. A. Kratzer and W. Franz, Transzendente Funktionen, (Geest \& Portig, Leipzig, 1960).

88. E.W. Barnes, Quarterly Journal of Mathematics 41 (1910) 136-140.

89. H. Mellin, Math. Ann. 68, no. 3 (1910) 305-337.

90. V.A. Smirnov, Feynman Integral Calculus, (Springer, Berlin, 2006).

91. M. Czakon, Comput. Phys. Commun. 175 (2006) 559-571, [hep-ph/0511200]

92. A. Smirnov and V. Smirnov, Eur. Phys. J. C62 (2009) 445-449, [arXiv: 0901.0386 [hep-ph]].

93. J. Gluza, K. Kajda, and T. Riemann, Comput. Phys. Commun. 177 (2007) 879-893, [arXiv:0704.2423[hep-ph]].

94. J. Gluza, K. Kajda, T. Riemann, and V. Yundin, Eur. Phys. J. C71 (2011) 1516, [arXiv:1010.1667[hep-ph]]

95. J. Ablinger, J. Blümlein, A. De Freitas, C. Schneider, and K. Schönwald, Nucl. Phys. B927 (2018) 339-367, [arXiv: 1711.06717 [hep-th]]

96. E.E. Kummer, J. Reine Angew. Math. (Crelle) 21 (1840) 74-90; 193-225; 328 371.

97. H. Poincaré, Acta Math. 4 (1884) 201-312.

98. J.A. Lappo-Danilevsky, Mémoirs sur la Théorie des Systèmes Différentielles Linéaires, (Chelsea Publ. Co, New York, 1953).

99. K.T. Chen, Trans. A.M.S. 156 (3) (1971) 359-379.

100. A.B. Goncharov, Math. Res. Lett. 5 (1998) 497-516.

101. A. von Manteuffel, E. Panzer, and R.M. Schabinger, JHEP 02 (2015) 120, [arXiv:1411.7392[hep-ph]].

102. F. Brown, Commun. Math. Phys. 287 (2009) 925-958, [arXiv:0804.1660 [math.AG]].

103. E. Panzer, Comput. Phys. Commun. 188 (2015) 148-166, [arXiv:1403.3385[hep-th]].

104. J. Ablinger, J. Blümlein, C. Raab, C. Schneider, and F. Wißbrock, Nucl. Phys. B885 (2014) 409-447, [arXiv:1403.1137[hep-ph]] 
105. F.Ph. Wißbrock, $O\left(\alpha_{s}^{3}\right)$ contributions to the heavy flavor Wilson coefficients of the structure function $F_{2}\left(x, Q^{2}\right)$ at $Q^{2} \gg m^{2}$, Ph.D. Thesis, TU Dortmund, 2015.

106. A.V. Kotikov, Phys. Lett. B254 (1991) 158-164.

107. Z. Bern, L.J. Dixon, and D.A. Kosower, Phys. Lett. B302 (1993) 299-308, [Erratum: Phys. Lett. B318, (1993) 649], [hep-ph/9212308]

108. E. Remiddi, Nuovo Cim. A110 (1997) 1435-1452, [hep-th/9711188]

109. T. Gehrmann and E. Remiddi, Nucl. Phys. B580 (2000) 485-518, [hep-ph/9912329]

110. S.A. Abramov and M. Petkovšek, D'Alembertian solutions of linear differential and difference equations, Proc. ISSAC'94, 169-174, ed. J. von zur Gathen, (ACM Press, 1994).

111. J. Ablinger, PoS (RADCOR2017) 001 [arXiv:1801.01039 [cs.SC]].

112. M.F. Singer, American Journal of Mathematics, 103 (4) (1981) 661-682.

113. J.J. Kovacic, J. Symb. Computation 2 (1986) 3-43.

114. J. Blümlein, J. Ablinger, A. Behring, A. De Freitas, A. von Manteuffel, C. Schneider and C. Schneider, PoS (QCDEV2017) 031 [arXiv:1711.07957 [hep$\mathrm{ph}]$.

115. J. Ablinger, A. Behring, J. Blümlein, G. Falcioni, A. De Freitas, P. Marquard, N. Rana, and C. Schneider, Phys. Rev. D 97 (2018) no.9, 094022 [arXiv:1712.09889 [hep-ph]].

116. J. Ablinger, J. Blümlein, P. Marquard, N. Rana and C. Schneider, Nucl. Phys. B 939 (2019) 253-291 [arXiv:1810.12261 [hep-ph]].

117. A.V. Kotikov, The Property of maximal transcendentality in the $N=4$ Supersymmetric Yang-Mills, In Subtleties in quantum field theory, ed. D. Diakonov, p. 150-174, [arXiv:1005.5029 [hep-th]].

118. J.M. Henn, Phys. Rev. Lett. 110 (2013) 251601, [arXiv:1304.1806[hep-th]].

119. J.M. Henn, J. Phys. A48 (2015) 153001, [arXiv:1412.2296[hep-ph]]

120. V.E. Zakharov, S.V. Manakov, S.P. Novikov, L.P. Pitaevskii, Teoria Solitonov: metod obratnoi zadatschi, (Nauka, Moskva, 1980).

121. S.Yu. Sakovich, J. Phys. A: Math. Gen. 28 (1995) 2861-2869.

122. R.N. Lee, JHEP 04 (2015) 108, [arXiv:1411.0911[hep-ph]].

123. M. Prausa, Comput. Phys. Commun. 219 (2017) 361-376, [arXiv:1701.00725 [hep-ph]]

124. O. Gituliar and V. Magerya, Comput. Phys. Commun. 219 (2017) 329-338, [arXiv:1701.04269[hep-ph]]

125. C. Meyer, Comput. Phys. Commun. 222 (2018) 295-312, [arXiv:1705.06252 [hep-ph]]

126. J. Ablinger, A. Behring, J. Blümlein, A. De Freitas, A. Hasselhuhn, A. von Manteuffel, M. Round, C. Schneider, and F. Wißbrock, Nucl. Phys. B886 (2014) 733-823, [arXiv:1406.4654[hep-ph]]

127. J. Ablinger, A. Behring, J. Blümlein, A. De Freitas, A. von Manteuffel, and C. Schneider, Nucl. Phys. B890 (2014) 48-151, [arXiv:1409.1135[hep-ph]]

128. J. Henn, A. V. Smirnov, V. A. Smirnov, and M. Steinhauser, JHEP 01 (2017) 074, [arXiv:1611.07535[hep-ph]]

129. J. Blümlein and C. Schneider, Phys. Lett. B771 (2017) 31-36, [arXiv: 1701.04614 [hep-ph]].

130. S.G. Gorishnii, S.A. Larin, L.R. Surguladze, F.V. Tkachov, Comput. Phys. Commun. 55 (1989) 381-408;

S.A. Larin, F.V. Tkachov and J.A.M. Vermaseren, The FORM version of $M I N-$ CER, NIKHEF-H-91-18.

131. M. Steinhauser, Comput. Phys. Commun. 134 (2001) 335-364 [hep-ph/ 0009029].

132. R. Harlander, T. Seidensticker, and M. Steinhauser, Phys. Lett. B426 (1998) 125-132, [hep-ph/9712228] 
133. T. Seidensticker, Automatic application of successive asymptotic expansions of Feynman diagrams, in: Proc. 6th International Workshop on New Computing Techniques in Physics Research, Crete, Greece, April 1999, hep-ph/9905298.

134. N.E. Nörlund, Vorlesungen über Differenzenrechnung, (Springer, Berlin, 1924); reprinted by (Chelsea Publishing Company, New York, 1954).

135. S.A. Larin, T. van Ritbergen, and J.A.M. Vermaseren, Nucl. Phys. B427 (1994) $41-52$.

136. S.A. Larin, P. Nogueira, T. van Ritbergen, and J.A.M. Vermaseren, Nucl. Phys. B492 (1997) 338-378, [hep-ph/9605317]

137. A. Retey and J.A.M. Vermaseren, Nucl. Phys. B604 (2001) 281-311, [hep-ph/0007294]

138. J. Blümlein and J.A.M. Vermaseren, Phys. Lett. B606 (2005) 130-138, [hep-ph/0411111]

139. I. Bierenbaum, J. Blümlein, and S. Klein, Nucl. Phys. B820 (2009) 417-482, [arXiv:0904.3563[hep-ph]]

140. J.A.M Vermaseren, A. Vogt, and S. Moch, Nucl. Phys. B724 (2005) 3-182, [hep-ph/0504242]

141. M. Kauers, M. Jaroschek, and F. Johansson, in: Computer Algebra and Polynomials, Editors: J. Gutierrez, J. Schicho, Josef, M. Weimann, Lecture Notes in Computer Science 8942 (Springer, Berlin, 2015) 105-125, [arXiv:1306.4263 [cs.SC]].

142. Sage, http://www. sagemath.org/.

143. J. Blümlein, M. Kauers, S. Klein, and C. Schneider, Comput. Phys. Commun. 180 (2009) 2143-2165, [arXiv:0902.4091[hep-ph]].

144. S. Moch, J.A.M. Vermaseren, and A. Vogt, Nucl. Phys. B688 (2004) 101-134, [hep-ph/0403192]

145. A. Vogt, S. Moch, and J.A.M. Vermaseren, Nucl. Phys. B691 (2004) 129-181, [hep-ph/0404111]

146. J. Ablinger, A. Behring, J. Blümlein, A. De Freitas, A. von Manteuffel, and C. Schneider, Nucl. Phys. B922 (2017) 1-40, [arxiv:1705.01508[hep-ph]].

147. J. Ablinger, J. Blümlein, P. Marquard, N. Rana and C. Schneider, Phys. Lett. B 782 (2018) 528-532 [arXiv:1804.07313 [hep-ph]].

148. G. Almkvist and D. Zeilberger, J. Symb. Comp. 10 (1990) 571-591.

149. M. Apagodu and D. Zeilberger, Adv. Appl. Math. (Special Regev Issue), 37 (2006) 139-152.

150. J. Ablinger, Computer Algebra Algorithms for Special Functions in Particle Physics, Ph.D. Thesis, Linz U. (2012) arXiv:1305.0687[math-ph].

151. D.J. Broadhurst, J. Fleischer, and O. V. Tarasov, Z. Phys. C60 (1993) 287-302, [hep-ph/9304303]

152. S. Bloch and P. Vanhove, J. Number Theor. 148 (2015) 328-364, [hep-th/ 1309.5865].

153. S. Laporta and E. Remiddi, Nucl. Phys. B704 (2005) 349-386, [hep-ph/0406160]

154. L. Adams, C. Bogner, and S. Weinzierl, J. Math. Phys. 54 (2013) 052303, [hep-ph/1302.7004]

155. L. Adams, C. Bogner, and S. Weinzierl, J. Math. Phys. 55 (2014), no. 10102301 , [hep-ph/1405.5640]

156. L. Adams, C. Bogner, and S. Weinzierl, J. Math. Phys. 56 (2015), no. 7072303 , [hep-ph/1504.03255]

157. L. Adams, C. Bogner, and S. Weinzierl, J. Math. Phys. 57 (2016), no. 3 032304, [hep-ph/1512.05630]

158. A. Sabry, Nucl. Phys. 33 (1962) 401-430.

159. E. Remiddi and L. Tancredi, Nucl. Phys. B907 (2016) 400-444, [arXiv:1602.01481[hep-ph]] 
160. L. Adams, C. Bogner, A. Schweitzer, and S. Weinzierl, J. Math. Phys. 57 (2016), no. 12 122302, [hep-ph/1607.01571].

161. J. Ablinger, J. Blümlein, A. De Freitas, M. van Hoeij, E. Imamoglu, C.G. Raab, C. S. Radu and C. Schneider, J. Math. Phys. 59 (2018) no.6, 062305 [arXiv: 1706.01299 [hep-th]].

162. J. Grigo, J. Hoff, P. Marquard and M. Steinhauser, Nucl. Phys. B 864 (2012) 580-596 [arXiv:1206.3418 [hep-ph]].

163. J. Blümlein, A. De Freitas, M. Van Hoeij, E. Imamoglu, P. Marquard and C. Schneider, PoS LL 2018 (2018) 017 [arXiv:1807.05287 [hep-ph]].

164. A. Ronveaux, ed., Heun's differential equations, (The Clarendon Press Oxford, Oxford, 1995).

165. E. Imamoglu and M. van Hoeij, J. Symbolic Comput. 83 (2017) 245-271, [arXiv:1606.01576 [cs.SC]].

166. K. Takeuchi, J. Fac. Sci, Univ. Tokyo, Sect. 1A 24 (1977) 201-272.

167. F.G. Tricomi, Elliptische Funktionen, (Geest \& Portig, Leipzig, 1948); übersetzt und bearbeitet von M. Krafft.

168. E.T. Whittaker and G.N. Watson, A Course of Modern Analysis, (Cambridge University Press, Cambridge, 1996), reprint of 4th edition (1927).

169. S. Herfurtner, Math. Ann. 291 (1991) 319-342.

170. H. Movasati and S. Reiter, Bull. Braz. Math Soc. 43 (2012) 423-442 [arXiv: 0902.0760[math.AG]].

171. J. Blümlein, talks at: The 5th International Congress on Mathematical Software ZIB Berlin from July 11 to July 14, 2016, Session: Symbolic computation and elementary particle physics, https://www.risc.jku.at/conferences/ICMS2016/; and QCD@LHC2016, U. Zürich, August 22 to August 26, 2016, https://indico.cern.ch/event/516210/timetable/\#all.detailed.

172. E. Remiddi and L. Tancredi, Nucl. Phys. B925 (2017) 212-251, [arXiv: 1709.03622[hep-ph]].

173. L. Adams and S. Weinzierl, Phys. Lett. B781 (2018) 270-278, [arXiv: 1802. 05020[hep-ph]].

174. J.-P. Serre, A Course in Arithmetic, (Springer, Berlin, 1973).

175. H. Cohen and F. Strömberg, Modular Forms, A Classical Approach, Graduate Studies in Mathematics 179 (AMS, providence, RI, 2017).

176. K. Ono, The Web of Modularity: Arithmetic of the Coefficients of Modular Forms and $q$-series, CBMS Regional Conference Series in Mathematics, 102 (AMS, Providence, RI, 2004).

177. H.H. Chan and W. Zudilin, Mathematika 56 (2010) 107-117.

178. D.J. Broadhurst, Eta quotients, Eichler integrals and L-series, talk at HMI Bonn, February 2018.

179. J. Brödel, C. Duhr, F. Dulat, B. Penante and L. Tancredi, JHEP 1808 (2018) 014 [arXiv:1803.10256 [hep-th]].

180. L. Adams and S. Weinzierl, Commun. Num. Theor. Phys. 12 (2018) 193-251 [arXiv:1704.08895 [hep-ph]].

181. C. Bogner, A. Schweitzer and S. Weinzierl, Nucl. Phys. B 922 (2017) 528-550 [arXiv:1705.08952 [hep-ph]].

182. J. Ablinger, J. Blümlein, A. De Freitas, A. Goedicke, C. Schneider and K. Schönwald, Nucl. Phys. B 932 (2018) 129-240 [arXiv:1804.02226 [hep-ph]].

183. J. Blümlein, A. De Freitas, C.G. Raab and K. Schönwald, Phys. Lett. B 791 (2019) 206-209 [arXiv:1901.08018 [hep-ph]].

184. J. Blümlein, A. De Freitas, C.G. Raab and K. Schönwald, The unpolarized two-loop massive pure singlet Wilson coefficients for deep-inelastic scattering, arXiv:1903.06155 [hep-ph].

185. J. Blümlein, C.G. Raab and K. Schönwald, The Polarized Two-Loop Massive Pure Singlet Wilson Coefficient for Deep-Inelastic Scattering, arXiv:1904.08911 [hep-ph]. 
186. M. Besier, D. Van Straten and S. Weinzierl, arXiv:1809.10983 [hep-th].

187. C.G. Raab. On the arithmetic of d'Alembertian functions. in preparation.

188. Li Guo, G. Regensburger, and M. Rosenkranz. J. Pure and Applied Algebra 218 (2014) 456-473.

189. J. Ablinger, J. Blümlein, C.G. Raab, and C. Schneider, J. Math. Phys. 55 (2014) 112301, [arXiv: 1407.1822 [hep-th]]

190. M.E. Hoffman, J. Algebraic Combin. 11 (2000) 49-68 [arXiv:math/9907173 [math.QA]].

191. J. Blümlein, Comput. Phys. Commun. 159 (2004) 19-54, [hep-ph/0311046].

192. J. Vermaseren, Int. J. Mod. Phys. A14 (1999) 2037-2076, [hep-ph/9806280].

193. J. Blümlein and S. Kurth, Phys. Rev. D60 (1999) 014018, [hep-ph/9810241].

194. J. Ablinger, J. Blümlein, and C. Schneider, J. Math. Phys. 52 (2011) 102301, [arXiv: 1105.6063 [math-ph]]

195. J. Ablinger, J. Blümlein, and C. Schneider, J. Math. Phys. 54 (2013) 082301, [arXiv: 1302.0378 [math-ph]]

196. A. I. Davydychev and M. Yu. Kalmykov, Nucl. Phys. B699 (2004) 3-64, [hep-th/0303162]

197. S. Weinzierl, J. Math. Phys. 45 (2004) 2656-2673, [hep-ph/0402131].

198. C. Reutenauer, Free Lie Algebras (Calendron Press, Oxford, 1993).

199. N. Nielsen Nova Acta Leopold. XC (1909) Nr. 3, 125-211.

200. K.S. Kölbig, J.A. Mignoco and E. Remiddi, BIT 10 (1970) 38-74.

201. K.S. Kölbig, SIAM J. Math. Anal. 17 (1986) 1232-1258.

202. A. Devoto and D.W. Duke, Riv. Nuovo Cim. 7N6 (1984) 1-39.

203. L. Lewin, Dilogarithms and associated functions, (Macdonald, London, 1958).

204. L. Lewin, Polylogarithms and associated functions, (North Holland, New York, 1981).

205. E. Remiddi and J.A.M. Vermaseren, Int. J. Mod. Phys. A15 (2000) 725-754, $[$ hep-ph/9905237]

206. S. Moch, P. Uwer, and S. Weinzierl, J. Math. Phys. 43 (2002) 3363-3386, [hep-ph/0110083]

207. S. Laporta, Phys. Lett. B772 (2017) 232-238, [arXiv:1704.06996[hep-ph]]

208. J. Blümlein and A. Vogt, Phys. Rev. D 58 (1998) 014020 [hep-ph/9712546].

209. J. Blümlein, V. Ravindran and W.L. van Neerven, Nucl. Phys. B 586 (2000) 349-381 [hep-ph/0004172].

210. J. Blümlein and A. Guffanti, Nucl. Phys. Proc. Suppl. 152 (2006) 87-91 [hep$\mathrm{ph} / 0411110]$.

211. J. Blümlein, Comput. Phys. Commun. 133 (2000) 76-104, [hep-ph/0003100]

212. J. Blümlein and S.-O. Moch, Phys. Lett. B614 (2005) 53-61, [hep-ph/0503188].

213. N. Nielsen, Handbuch der Theorie der Gammafunktion, (Teubner, Leipzig, 1906); reprinted by.(Chelsea Publishing Company, Bronx, New York, 1965).

214. E. Landau, Über die Grundlagen der Theorie der Fakultätenreihen, S.-Ber. math.naturw. Kl. Bayerische Akad. Wiss. München, 36 (1906) 151-218.

215. J. Blümlein, Clay Math. Proc. 12 (2010) 167-188, [arXiv:0901.0837[math-ph]].

216. J. Blümlein, Comput. Phys. Commun. 180 (2009) 2218-2249, [arXiv:0901.3106 [hep-ph]]

217. A.V. Kotikov and V.N. Velizhanin, Analytic continuation of the Mellin moments of deep inelastic structure functions, hep-ph/0501274.

218. J. Ablinger, PoS (LL2014) 019, [arXiv:1407.6180[cs.SC]]].

219. J. Ablinger, A Computer Algebra Toolbox for Harmonic Sums Related to Particle Physics, Diploma Thesis, JKU Linz, 2009, arXiv:1011.1176[math-ph].

220. S. Weinzierl, Comput. Phys. Commun. 145 (2002) 357-370, [math-ph/0201011].

221. S. Moch and P. Uwer, Comput. Phys. Commun. 174 (2006) 759-770, [math-ph/0508008]

222. C. Duhr and F. Dulat, PolyLog Tools - Polylogs for the masses, arXiv:1904.07279 [hep-th]. 
223. T. Gehrmann and E. Remiddi, Comput. Phys. Commun. 141 (2001) 296-312, [hep-ph/0107173]

224. D. Maitre, Comput. Phys. Commun. 174 (2006) 222-240, [hep-ph/0507152]

225. J. Ablinger, J. Blümlein, M. Round, and C. Schneider, PoS (RADCOR2017) 010 [arXiv:1712.08541[hep-th]].

226. J. Ablinger, J. Blmlein, M. Round and C. Schneider, Numerical Implementation of Harmonic Polylogarithms to Weight $w=8$, Comp. Phys. Commun. (2018) in print, arXiv:1809.07084 [hep-ph].

227. J. Vollinga and S. Weinzierl, Comput. Phys. Commun. 167 (2005) 177, $[$ hep-ph/0410259]

228. C. Neumann, Vorlesungen über Riemann's Theorie der Abel'schen Integrale, (Teubner, Leipzig, 1884), 2nd Edition.

229. F. Brown and O. Schnetz, Duke Math. Journal 161 No. 10 (2012) 1817-1862.

230. D. Hilbert, Grundzüge einer allgemeinen Theorie der linearen Integralgleichungen, (Teubner, Leipzig, 1912).

231. R. de L. Kronig, J. Opt. Soc. Am. 12 (1926) 547-557.

232. H.A. Kramers, Atti Cong. Intern. Fisici, (Transactions of Volta Centenary Congress) Como, 2 (1927)

233. S. Abreu, R. Britto, C. Duhr, and E. Gardi, JHEP 12 (2017) 090, [arXiv:1704.07931[hep-th]]

234. M.J.G. Veltman, Physica 29 (1963) 186-207.

235. E. Remiddi, Helv. Phys. Acta 54 (1982) 364-382;

E. Remiddi, Differential Equations and Dispersion Relations for Feynman Amplitudes, in: Elliptic Integrals, Elliptic Functions and Modular Forms in Quantum Field Theory, eds. J. Blümlein, C. Schneider, and P. Paule, (Springer, Wien, 2019), 391-414. 